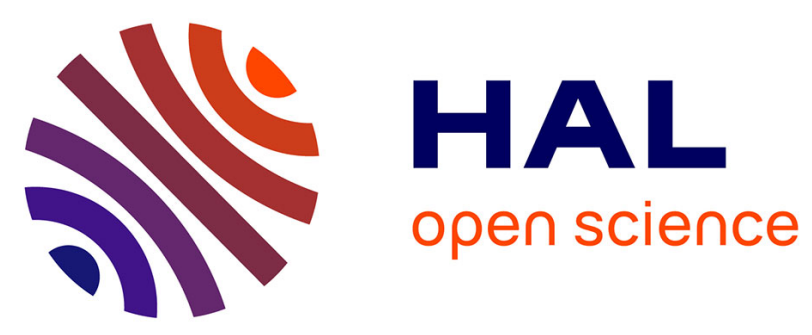

\title{
Excited State Dynamics of Protonated Phenylalanine and Tyrosine: Photo-Induced Reactions Following Electronic Excitation
}

Géraldine Féraud, Michel Broquier, Claude Dedonder, Christophe Jouvet, Gilles Grégoire, Satchin Soorkia

\section{To cite this version:}

Géraldine Féraud, Michel Broquier, Claude Dedonder, Christophe Jouvet, Gilles Grégoire, et al.. Excited State Dynamics of Protonated Phenylalanine and Tyrosine: Photo-Induced Reactions Following Electronic Excitation. Journal of Physical Chemistry A, 2015, 119, pp.5914. 10.1021/jp5065837. hal-01164711

\author{
HAL Id: hal-01164711 \\ https://hal.science/hal-01164711
}

Submitted on 18 Jun 2015

HAL is a multi-disciplinary open access archive for the deposit and dissemination of scientific research documents, whether they are published or not. The documents may come from teaching and research institutions in France or abroad, or from public or private research centers.
L'archive ouverte pluridisciplinaire HAL, est destinée au dépôt et à la diffusion de documents scientifiques de niveau recherche, publiés ou non, émanant des établissements d'enseignement et de recherche français ou étrangers, des laboratoires publics ou privés. 


\title{
Excited-State Dynamics of Protonated Phenylalanine and Tyrosine: Photo- Induced Reactions Following Electronic Excitation
}

\author{
Géraldine Féraud, ${ }^{1}$ Michel Broquier, ${ }^{2,3}$ Claude Dedonder, ${ }^{1}$ Christophe Jouvet, ${ }^{1}$ Gilles \\ Grégoire, ${ }^{4 *}$ Satchin Soorkia ${ }^{3}$
}

1) CNRS, Aix Marseille Université, laboratoire de Physique des Interactions Ioniques et Moléculaires (PIIM) UMR 7345, 13397 Marseille cedex 20, France

2) Université Paris Sud, CLUPS (Centre Laser de l'Université Paris Sud) LUMAT FR 2764, 91405 Orsay Cedex, France

3) CNRS, Université Paris Sud, Institut des Sciences Moléculaires d'Orsay (ISMO) UMR 8624, 91405 Orsay Cedex, France

4) CNRS, Université Paris 13, Sorbonne Paris Cité, Laboratoire de Physique des Lasers, UMR 7538, 93430 Villetaneuse, France.

* Corresponding author: E-mail : gilles.gregoire@univ-paris13.fr

\begin{abstract}
The electronic spectroscopy and the electronic excited state properties of cold protonated phenylalanine and protonated tyrosine have been revisited on a large spectral domain and interpreted by comparison with ab initio calculations. The protonated species are stored in a cryogenically cooled Paul trap, maintained at $\sim 10 \mathrm{~K}$, and the parent and all the photo-fragment ions are mass-analyzed in a time-of-flight mass spectrometer, which allows detecting the ionic species with an improved mass resolution compared to what is routinely achieved with a quadrupole mass spectrometer. These new results emphasize the competition around the band origin between two proton transfer reactions from the ammonium group toward either the aromatic chromophore or the carboxylic acid group. These reactions are initiated by the coupling of the locally excited $\pi \pi^{*}$ state with higher charge transfer states, the positions and coupling of which depend on the conformation of the protonated molecules. Each of these reaction processes gives rise to specific fragmentation channels that supports the conformer selectivity observed in the photofragmentation spectra of protonated Tyrosine and Phenylalanine.
\end{abstract}

Keywords:

Photo-fragmentation spectroscopy; protonated aromatic amino acid; cold ion; proton transfer reaction; charge transfer state; ab initio calculation. 


\section{Introduction}

Gas phase spectroscopy of the neutral aromatic amino acids phenylalanine, ${ }^{1,2}$ tyrosine $^{1,3-6}$ and tryptophan $^{7-9}$ in molecular beams have been extensively documented over the last decades. In contrast, the protonated analogues have been studied very recently. ${ }^{10-12}$ The coupling of an electrospray source with a cold ion trap has contributed to the ease of production and spectroscopic studies of such species. An important milestone was reached in the seminal work of O. Boyarkin and T.R. Rizzo in Lausanne ${ }^{11-13}$ using a cold 22-pole ion trap developed by D. Gerlich. ${ }^{14}$ Since then, several experimental set-ups using this technique ${ }^{15-17}$ have been built and a simplified version has been developed using a Paul ion trap. ${ }^{18-22}$

The UV photofragmentation spectra of protonated phenylalanine $\left(\mathrm{PheH}^{+}\right)$and tyrosine $\left(\mathrm{TyrH}^{+}\right)$ have already been recorded ${ }^{12}$ on a limited spectral domain, and show well-resolved vibronic bands. For both protonated species, two low-lying conformers, which differ by $\sim 2 \pi / 3$ rotation of the carboxylic acid group along the $\mathrm{C}_{\alpha}-\mathrm{C}_{\beta}$ bond, have been assigned using IR-UV double resonance spectroscopy and comparison with DFT calculations. In the case of $\mathrm{TyrH}^{+}$, each of these two conformations can adopt an anti or syn orientation of the hydroxyl group of the phenyl chromophore. One of the striking results of these photofragmentation experiments was the observation of specific fragmentation channels as compared to the typical fragmentation spectrum obtained through collision-induced dissociation experiments. ${ }^{23}$ Around the band origin of the electronic excitation, the two protonated molecules mostly fragment through the $\mathrm{C}_{\alpha}-\mathrm{C}_{\beta}$ bond rupture. ${ }^{12}$ For $\mathrm{PheH}^{+}$, this is the only fragmentation pathway leading to the detection of $\mathrm{m} / \mathrm{z} 74 \pm 1$ and $92 \pm 1$ depending on the localization of the charge on the final product. It should be reminded that in the first studies of the Rizzo's group, ${ }^{12}$ the detection scheme used a quadrupole mass analyzer that had a low mass-resolution and the exact mass of the product ions could not be accurately determined, within \pm 1 mass units. In the case of $\mathrm{TyrH}^{+}, \mathrm{C}_{\alpha}-\mathrm{C}_{\beta}$ bond breaking is evidenced by the detection of $\mathrm{m} / \mathrm{z} 107 \pm 1$ along with minor CID-like fragments at $\mathrm{m} / \mathrm{z} 147,136,123$ and 119, which account for less than $10 \%$ of the overall fragmentation yield. Higher massresolution experiments showed that the $\mathrm{m} / \mathrm{z} 107 \pm 1$ fragment is in fact the protonated tyrosine side chain at $\mathrm{m} / \mathrm{z} 108 .{ }^{17,24}$ Besides, it has been shown that the fragmentation channels in $\mathrm{TyrH}^{+}$are conformer-dependent but this behavior has not yet been elucidated. This latter result strongly suggests that the fragmentation mechanisms are governed by complex dynamical processes in the electronic excited states. In this paper, we investigate and decipher the influence of the conformation of the molecules upon their fragmentation pathways and the evolution with the excess energy in the locally excited state. 
The fragmentation time is also very important information that can help to differentiate fragmentations occurring in the excited states (very fast sub nanosecond) from processes occurring after internal conversion in the ground state or secondary fragmentations (i.e. dissociation of hot fragments). The different processes have been fully characterized for protonated tryptophan $\left(\operatorname{TrpH} \mathrm{H}^{+}\right)$ using the ion beam ion/neutral coincidence experiment ${ }^{25-28}$ for fragmentation processes and pump/probe femtosecond experiment for fast dynamics in the excited state. ${ }^{29}$ These experiments combined with $a b$ initio calculations provide a complete picture of the $\mathrm{TrpH}^{+}$fragmentation following excitation at $266 \mathrm{~nm}$ and a relationship between the excited state and the fragmentation mechanism has been proposed. Less information has been obtained on $\mathrm{PheH}^{+}$and $\mathrm{TyrH}^{+}$due to their lower oscillator strengths at $266 \mathrm{~nm}$. For TyrH ${ }^{+}$the excited state lifetime is short (22 ps at 266 $\mathrm{nm}$ ) but longer than the $\operatorname{TrpH}^{+}$lifetime, which exhibits a bi-exponential decay with time constants of $400 \mathrm{fs}$ and 15 ps. $^{29,30}$

From a theoretical point of view, the excited state properties of neutral amino acids ${ }^{31-33}$ and protonated species ${ }^{34-36}$ have been investigated. For the protonated species, these calculations have shown that three low-lying excited states play a crucial role. They correspond to the promotion of an electron from the HOMO, which is a $\pi$ orbital localized on the aromatic part, to three different unoccupied orbitals. The first one is the $\pi^{*}$ orbital localized on the aromatic ring and the $\pi \pi^{*}$ state is responsible for the optical absorption and carries the oscillator strength (for $\operatorname{TrpH}^{+}$there are two $\pi \pi^{*}$ states so-called $\mathrm{L}_{\mathrm{a}}$ and $\left.\mathrm{L}_{\mathrm{b}}\right){ }^{37}$ The second important orbital is a Rydberg-type orbital localized on the protonated amino group. This orbital has $\sigma$ symmetry with respect to the glycine plane $\left(\sigma_{\mathrm{NH} 3}{ }^{*}\right)$. The third one is mainly localized on the carbonyl group and has $\pi$ type symmetry with respect to the glycine plane $\left(\pi_{\mathrm{CO}} *\right)$. The energy gap between the locally excited $\pi \pi^{*}$ state and the higher excited states of $\pi \sigma^{*}$ and $\pi \pi_{\mathrm{CO}} *$ characters is the smallest in $\mathrm{TrpH}^{+}$and increases for $\mathrm{TyrH}^{+}$ and $\mathrm{PheH}^{+}$. This variation of the energy gap can be understood with simple arguments: $:^{29,34,35}$ in essence, to reach these states, one electron has to be removed from the aromatic part and added to the protonated glycine part. The energies of these states then strongly depend on the ionization energy of the aromatic moiety, which is lowest for Trp, followed by Tyr and Phe. As a consequence, the electron transfer is more favorable in Trp than in Tyr or Phe. Ab initio calculations have confirmed this model: the energy gap between the $\pi \pi *$ state and the states localized on the glycine part is in the order of $0.3 \mathrm{eV}$ for $\mathrm{TrpH}^{+}$while it is $0.9 \mathrm{eV}$ in $\mathrm{TyrH}^{+}$and $1.1 \mathrm{eV}$ in $\mathrm{PheH}^{+}$. In the case of $\mathrm{TyrH}^{+}$, the direct excitation of the $\pi \sigma *$ state has been recently evidenced below $240 \mathrm{~nm}$, leading to the formation of the radical cation ( $\mathrm{H}$ loss channel), which subsequently fragments through the $\mathrm{C}_{\alpha}-\mathrm{C}_{\beta}$ bond dissociation forming $\mathrm{m} / \mathrm{z} 107$ fragment. ${ }^{38}$ For $\mathrm{PheH}^{+}$, calculation indicates that vertical 
excitation of the $\pi \sigma^{*}$ occurs at higher energy, more than $1 \mathrm{eV}$ above the origin transition of the $\pi \pi^{*}$ state and that cannot be reached with our UV laser.

In this paper we present new experimental results of the UV photodissociation of cold $\mathrm{PheH}^{+}$and $\mathrm{TyrH}^{+}$on a large spectral domain supported by high-level $a b$ initio calculations on the electronic excited states. The protonated species, produced with an ESI source, are guided and trapped in a cold quadrupole ion Paul trap $(10-15 \mathrm{~K})$. All ionic fragments and parent molecules are massanalyzed in a time-of-flight mass spectrometer, which allows detecting all the ionic species with very good mass-resolution. The present results emphasize the close relationship between the electronic character of the excited states and the fragmentation channels which is evidenced by the profound variation of the branching ratio of the fragmentation channels as a function of the excitation energy. Geometry optimizations of the different excited states have evidenced the role of electron transfer and proton transfer reactions in the UV photodissociation process. Besides, we propose an explanation for the dependence of the fragmentation channels on the conformation of the protonated aromatic amino acids.

\section{Experimental methods}

The electronic spectrum of protonated aromatic amino acids was obtained via photo-fragment spectroscopy in a cryogenic cold quadrupole ion trap (Paul ion trap from Jordan TOF Products, Inc.) installed in the Orsay laboratory (CLUPS). The setup is similar to the one developed by Wang and Wang. ${ }^{18,19}$ Gas-phase protonated species are produced in an electrospray ionization source built at Aarhus University. ${ }^{39}$ At the exit of the capillary, the ions are guided in an octopole and trapped by applying a positive voltage $(\sim 10 \mathrm{~V})$ on the exit electrode for $90 \mathrm{~ms}$. They are extracted by applying a negative voltage of $-30 \mathrm{~V}$ during $4 \mu \mathrm{s}$ and are further accelerated to $200 \mathrm{~V}$ by a second pulsed voltage just after the exit electrode. This time sequence of pulsed voltages produces ion packets with a duration time between $500 \mathrm{~ns}$ and $1 \mu \mathrm{s}$. The ions are driven by a couple of electrostatic lenses toward the Paul trap biased at a voltage close to the kinetic energy of the ions, which is defined by the second pulsed voltage at the exit of the octopole. This ensures that limited collisions-induced fragmentation occur when the ions enter the trap. A mass gate placed at the entrance of the trap allows selecting the parent ion. The Paul trap is mounted on the cold head of a cryostat connected to a water-cooled He compressor. The temperature of the trap is monitored by temperature diode-sensors: a first one directly mounted on the cold head of the cryostat $\left(\mathrm{T}_{\mathrm{a}}\right)$ and a second one $\left(\mathrm{T}_{\mathrm{b}}\right)$ mounted on top of the copper box which houses the trap. Typical temperatures are $\mathrm{T}_{\mathrm{a}}=9 \mathrm{~K}$ and $\mathrm{T}_{\mathrm{b}}=12 \mathrm{~K}$. Helium as buffer gas is injected in the trap using a pulsed valve (General Valve) triggered 1-2 ms before the ions enter the trap. The valve is opened for $150 \mu \mathrm{s}$ and the 
pressure in the vacuum chamber rises by $5-810^{-7}$ mbar. The ions are trapped and thermalized through collisions with buffer gas: the thermalization is reached after a trapping time of typically 3 $\mathrm{ms}^{19}$. The photodissociation laser is triggered after $30 \mathrm{~ms}$, and the parent and fragment ions are kept in the trap for $80 \mathrm{~ms}$ before extraction to the time-of-flight mass spectrometer. This delay ensures complete evacuation of the He buffer gas from the trap. In these conditions, no collision-induced fragmentation is observed when the ions are extracted and accelerated for analysis in the linear time of flight mass spectrometer of $1.0 \mathrm{~m}$ long. Photo-fragmentation spectra are obtained by recording the fragment ion signal on a microchannel plates (MCP) detector as a function of laser wavelength with a digitizing storage oscilloscope interfaced to a PC.

The photo-dissociation laser is a dye laser (Quantel TDL 90), which has a resolution of $\sim 0.2$ $\mathrm{cm}^{-1}$. For protonated phenylalanine, the UV radiation $(254-267 \mathrm{~nm})$ is produced by mixing the frequency-doubled output of the dye lasers with the fundamental radiation of the Nd:YAG pump laser (Quantel). For protonated tyrosine, the laser radiation is obtained by frequency doubling several dyes to cover the $275-285 \mathrm{~nm}$ region. All the recorded spectra have been obtained with energy from 10 to $100 \mu \mathrm{J} /$ pulse $\left(2-3 \mathrm{~mm}^{2}\right.$ collimated beam). For this typical energy of the laser, the fragmentation signal is on the order of $1-3 \%$ of the parent ion signal. The photo-fragmentation signal is normalized by the parent ion signal to account for the fluctuations of the electrospray ion source and corrected by the laser intensity.

\section{Computational methods}

$A b$ initio calculations have been performed with the TURBOMOLE program package (v6.2) ${ }^{40}$ making use of the resolution-of-the-identity (RI) approximation for the evaluation of the electron-repulsion integrals. ${ }^{41}$ The equilibrium geometries of protonated aromatic amino acids in their ground electronic $\left(\mathrm{S}_{0}\right)$ and excited states $\left(\mathrm{S}_{\mathrm{n}=1-4}\right)$ have been determined at the CC2 level. In construction of the reaction path for the proton transfer reaction from $\mathrm{NH}_{3}{ }^{+}$to the cycle, the coordinate-driven minimum-energy-path (MEP) approach was utilized, i.e. for a given N-H distance, all remaining intramolecular coordinates were optimized in the $S_{1}$ state. Calculations were performed with the correlation-consistent polarized valence double-zeta aug-cc-pVDZ basis set augmented with diffuse functions. ${ }^{42}$ The vibrational modes of the ground and the first excited states have been calculated at the same level and the Franck-Condon analysis have been performed using PGOPHER software. ${ }^{43}$ The NBO analysis ${ }^{44}$ has been performed as implemented in the G09 software. $^{45}$ 


\section{Results}

\section{Protonated Phenylalanine}

The figure 1 shows the vibrationnally resolved ultraviolet photo-fragmentation spectra from the origin of the electronic transition located at $37520.9 \mathrm{~cm}^{-1}$ and up to $1800 \mathrm{~cm}^{-1}$ above in energy. This spectrum is similar to the one recorded in the group of $\mathrm{T}$. Rizzo ${ }^{12}$ from the $\mathrm{S}_{1}$ origin band up to $800 \mathrm{~cm}^{-1}$ above in energy, in which two conformers $\mathrm{A}$ and $\mathrm{B}$, which differ by a $2 \pi / 3$ rotation around the $\mathrm{C}_{\alpha}-\mathrm{C}_{\beta}$ bond, were assigned. The conformer with both carbonyl and protonated amino groups lying above the aromatic ring will be denoted stack (conformer B in ref 12), and the conformer obtained by a $2 \pi / 3$ rotation along the $\mathrm{C}_{\alpha}-\mathrm{C}_{\beta}$ bond, with only the protonated amino group lying above the ring, will be denoted rot (conformer A in ref 12). In our study, the mass resolution of the time-of-flight mass spectrometer allows to firmly assign the fragmentation channels and their branching ratio as a function of the excess energy in the $S_{1}$ state. At low photon energies, only two photo-fragments at m/z 75 and m/z 92 are detected (see Supporting Information Figure SI1). These two fragments originate from the $\mathrm{C}_{\alpha}-\mathrm{C}_{\beta}$ bond rupture after proton transfer to the aromatic ring, leading to the formation of the $\mathrm{C}_{6} \mathrm{H}_{6}{ }^{+}-\mathrm{CH}_{2}$ radical cation that most probably rearranges into toluene ion $(\mathrm{m} / \mathrm{z} 92)$ or the glycine ion $(\mathrm{m} / \mathrm{z} 75)$ after back proton transfer to the amino acid fragment. The photo-fragmentation yield of $\mathrm{m} / \mathrm{z} 75$ fragment is greater than that of $\mathrm{m} / \mathrm{z} 92$ fragment by a factor of two in our experimental conditions.

In this low energy region, an intense vibronic band at $531 \mathrm{~cm}^{-1}$ above the band origins assigned to the excitation of the $6 \mathrm{~b}$ vibrational mode of benzene and its derivatives (in plane deformation of the benzene ring) ${ }^{46}$ is observed for the two conformers. From this excess energy above the origin transition, a new fragmentation channel at m/z 120 appears. This fragment results from the loss of $\mathrm{CO}+\mathrm{H}_{2} \mathrm{O}$, leading to the formation of the iminium ion of phenylalanine. The photofragmentation yield recorded on this fragment is null at the $S_{1}$ origin, very weak for the $6 b_{0}{ }^{1}$ band, and increases continuously afterwards while the vibrational progression observed on the $\mathrm{m} / \mathrm{z} 75$ and $\mathrm{m} / \mathrm{z} 92$ fragments disappears $630 \mathrm{~cm}^{-1}$ above the origin. It is noteworthy that the electronic spectrum of protonated phenylalanine still exhibits well-structured transitions more than $1500 \mathrm{~cm}^{-1}$ above the band origin. In particular, the same vibrational progression of low frequency modes observed from the origin and $6 b_{0}{ }^{1}$ bands shows up from the band at $38975 \mathrm{~cm}^{-1}$, i.e. $1454 \mathrm{~cm}^{-1}$ above the origin transition. This transition is assigned to the $6 \mathrm{~b}_{0}{ }^{1} 12_{0}{ }^{1}$ combination band based on the assignment of neutral $^{47}$ and protonated ${ }^{22}$ phenylethylamine. Note that the relative intensity of the $6 b_{0}{ }^{1} 12_{0}{ }^{1}$ combination band and the $6 \mathrm{~b}_{0}{ }^{1}$ band is greater for the rot conformer than for the stack conformer, at variance with what is observed at the band origin. The intensity of the origin transition assigned to the rot conformer is significantly smaller than the one of the stack structure with a ratio of 0.5 
which rises to 2.6 and 3.4 for the $6 \mathrm{~b}_{0}{ }^{1}$ band and the $6 \mathrm{~b}_{0}{ }^{1} 12_{0}{ }^{1}$ combination band, respectively. In conclusion, the electronic spectrum recorded up to $1800 \mathrm{~cm}^{-1}$ above the origin band reveals the $\pi^{*}$ character of the first excited state of protonated phenylalanine. The most striking result is the drastic evolution of the branching ratio of the different fragmentation channels and the relative intensity of the transitions assigned to the rot and stack conformers with the excess energy in the $\pi \pi^{*}$ state.

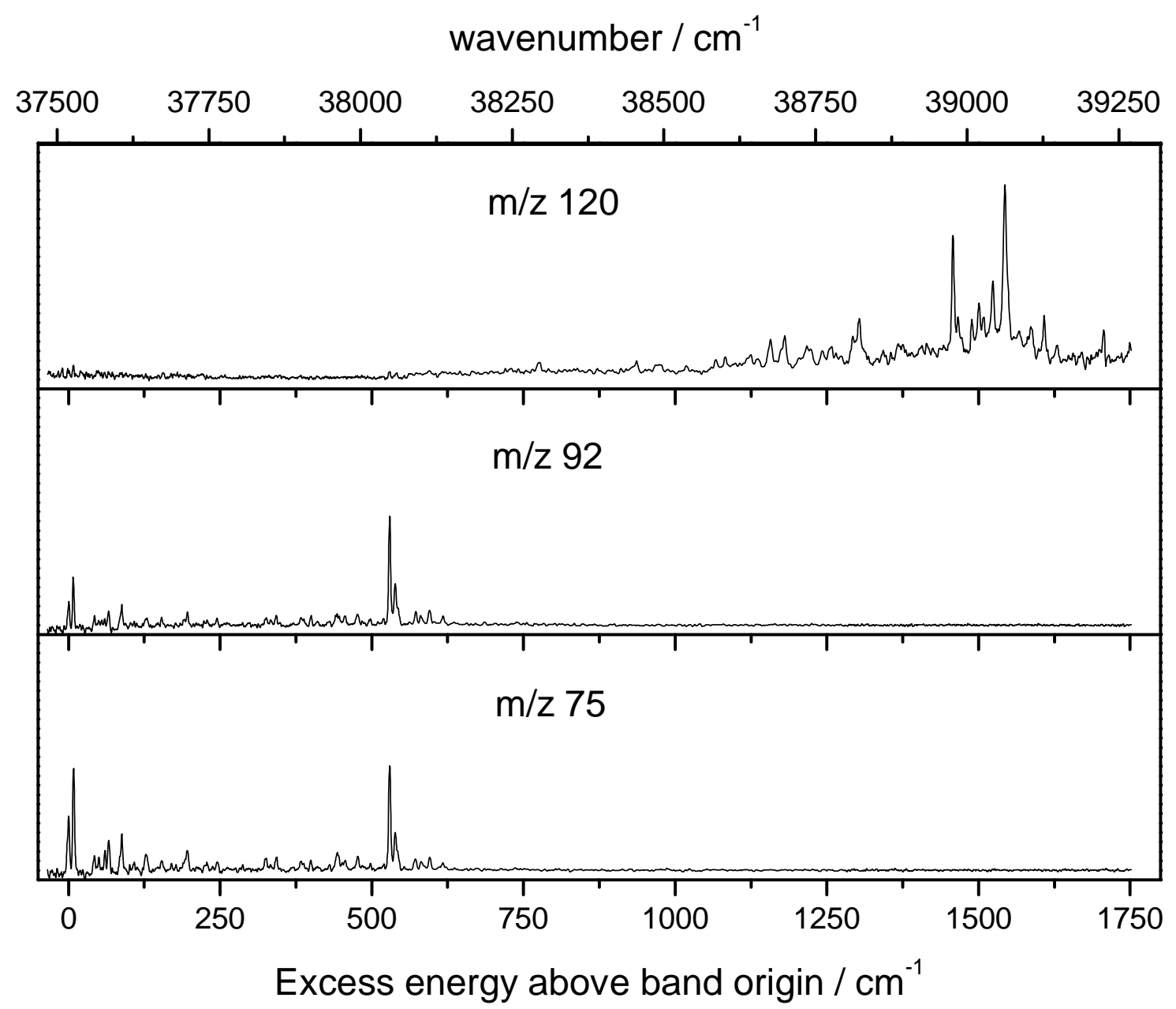

Figure 1: Photo-fragmentation spectra of protonated phenylalanine recorded on the three main fragmentation channels (same y axis scale) over a large spectral range: at low energies the fragmentation results in $\mathrm{m} / \mathrm{z} 75$ and $\mathrm{m} / \mathrm{z} 92$ following a proton transfer to the aromatic chromophore, while at higher energies these fragments disappear and $\mathrm{m} / \mathrm{z}$ 120 is the only fragment produced.

\section{Protonated Tyrosine}

The vibrationnally resolved electronic spectrum of protonated tyrosine up to $2000 \mathrm{~cm}^{-1}$ above the band origin is shown in figure 2. In the low frequency region, this spectrum is very similar to those obtained previously by Stearns et al. ${ }^{12}$ and more recently by Redwine et al. ${ }^{17}$ in a 22-pole trap. Four conformers have been previously assigned from IR/UV depletion spectroscopy, ${ }^{12}$ which differ from each other by a $2 \pi / 3$ rotation around the $C_{\alpha}-C_{\beta}$ bond (stack and rot as for phenylalanine) and the orientation of the hydroxyl group of the phenyl ring compared to the position of the protonated 
amino group. To distinguish the orientation of the $\mathrm{OH}$ group, we introduce the anti and syn labels that correspond to the localization of the hydroxyl lone pair relative to the protonated ammonium group. Stack-syn, rot-syn, stack-anti and rot-anti conformers thus correspond to A, B, C and D conformers of reference 12 , respectively.

As for phenylalanine, the fragmentation channels drastically change as a function of the excess energy. At the band origin, the main fragmentation channel is $\mathrm{m} / \mathrm{z} 108$, assigned to the $\mathrm{C}_{\alpha}-\mathrm{C}_{\beta}$ bond rupture after proton transfer from the ammonium group to the aromatic ring. This fragment is tenfold more intense than the other ones at m/z 147, m/z 136, m/z 123 and m/z 119. From $800 \mathrm{~cm}^{-1}$ above the band origins of the four conformers, the sum of the fragmentation yields of the $\mathrm{m} / \mathrm{z} 147$, $\mathrm{m} / \mathrm{z} 136, \mathrm{~m} / \mathrm{z} 123$ and $\mathrm{m} / \mathrm{z} 119$ becomes equal to the signal recorded on the $\mathrm{m} / \mathrm{z} 108$ fragment. Higher in energy, the fragmentation yield of the $\mathrm{m} / \mathrm{z} 108$ almost vanishes while the other fragments gain in intensity. Regardless of the number of conformers, the spectra recorded up to $2000 \mathrm{~cm}^{-1}$ above the band origin are similar to that of the neutral species, ${ }^{3-5}$ indicating that the electronic transition has a $\pi \pi^{*}$ character. For instance, the vibrational pattern located at $+810 \mathrm{~cm}^{-1}$ arises from the ring "breathing" mode, or mode 1, according to Wilson notation. The transitions seen at $+1600 \mathrm{~cm}^{-1}$ are assigned to the $1_{0}^{2}$ band.

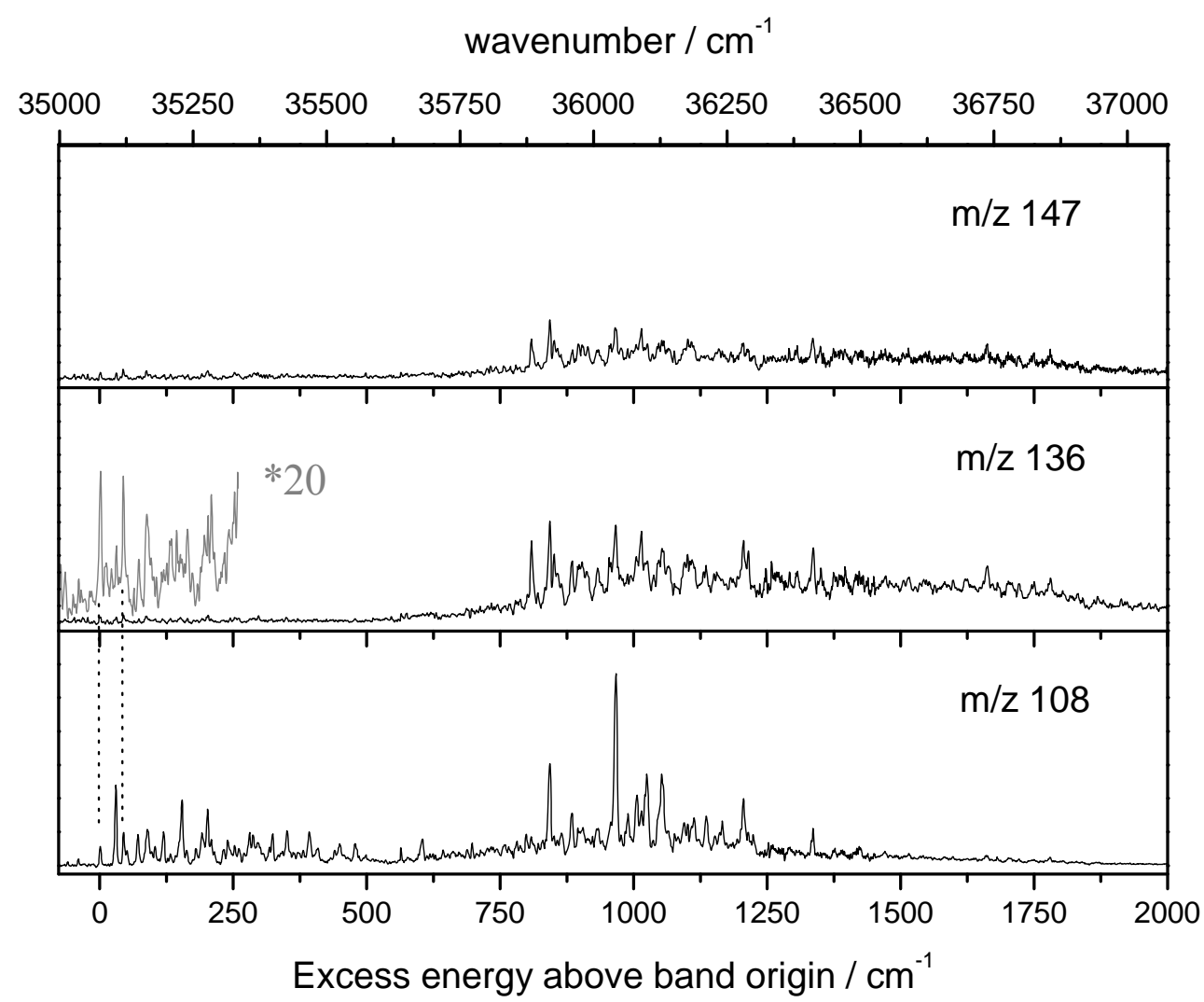

Figure 2: Photo-fragmentation spectra of protonated tyrosine recorded on the three main fragmentation channels (same y axis scale) over a large spectral range: at low energies the fragmentation results mainly in $\mathrm{m} / \mathrm{z} 108$, while at higher energies the $\mathrm{m} / \mathrm{z} 147$ and $\mathrm{m} / \mathrm{z} 136$ fragments become as intense as the former one. The spectra recorded on $\mathrm{m} / \mathrm{z}$ 123 and m/z 119 fragments are not presented but they are similar to m/z 136 and m/z 147.The vertical dotted lines show positions of the origin and the first vibronic band at $43 \mathrm{~cm}^{-1}$ of the stack-syn conformer. 
As previously reported by Stearns et $a l^{12}$ and Redwine et al. ${ }^{17}$, we do observe a clear dependence of the fragmentation channel upon the probed conformer. Around the origin, the rot conformers are mostly detected at $\mathrm{m} / \mathrm{z} 108$, while the stack conformers are barely observed. This is the inverse when looking at the spectra of other fragmentation channels, i.e. the transitions assigned to the rot conformers are almost absent. Around the $1_{0}{ }^{1}$ band at $+810 \mathrm{~cm}^{-1}$, the rot conformers are the only ones detected on the $\mathrm{m} / \mathrm{z} 108$ fragment, while all conformers exhibit vibronic transitions on the $\mathrm{m} / \mathrm{z}$ 147, m/z 136, m/z 123 and m/z 119 fragmentation channels. From the $1_{0}{ }^{1}$ band, the absence of transition assigned to the stack conformations on the fragmentation channel related to the $\mathrm{C}_{\alpha}-\mathrm{C}_{\beta}$ bond break (m/z 108) clearly reveals that this fragmentation channel is conformer-dependent, which suggests that the dynamics in the $\pi \pi^{*}$ excited state has changed drastically.

It should be noted that such a strong selectivity in the branching ratio of the fragmentation yield with the conformations of tyrosine is observed at low laser intensity. The photofragmentation yield follows a linear dependence as a function of the laser energy for low laser powers below 100 $\mu \mathrm{J} /$ pulse, while a two-photon absorption regime occurs above $100 \mu \mathrm{J} /$ pulse until saturation is reached from $200 \mu \mathrm{J} /$ pulse (Figure SI 3 of supplementary information).

For both molecules, the width of the vibronic bands does not change in the entire spectral range, being in the order of $5 \mathrm{~cm}^{-1}$, while the change in the fragmentation channels as a function of the excitation energy is noteworthy. For $\mathrm{PheH}^{+}$, the closing of $\mathrm{m} / \mathrm{z} 75$ and $\mathrm{m} / \mathrm{z} 92$ channels is correlated to the opening of the m/z 120 fragmentation channel. For $\mathrm{TyrH}^{+}$, the $\mathrm{m} / \mathrm{z} 108$ channel has the highest relative photofragmentation yield but is in competition with other fragmentation channels. It is worth mentioning that the $\mathrm{C}_{\alpha}-\mathrm{C}_{\beta}$ bond cleavage is a UV photo-specific fragmentation channel. The other fragmentation channels that appear with increasing excess energy in the first excited state are those which are detected in low-energy CID experiments. ${ }^{23}$ At low excess energy in the excited state, the $\mathrm{C}_{\alpha}-\mathrm{C}_{\beta}$ bond cleavage is the dominant fragmentation channel (and even unique in $\mathrm{PheH}^{+}$). The same trends and competition have been observed in the UV photofragmentation of protonated phenylethylamine and tyramine. ${ }^{22}$ In these simpler protonated aromatic amines, this competition has been rationalized in the frame of the $\pi \pi^{*}-\pi \sigma^{*}$ model. ${ }^{48}$ Around the band origin, proton transfer from the amino group to the aromatic ring is the dominant process in the excited state leading to the $\mathrm{C}_{\alpha}-\mathrm{C}_{\beta}$ bond cleavage. At higher excess energy in $\mathrm{S}_{1}$, the coupling of the $\pi \pi^{*}$ state with the $\pi \sigma^{*}$ charge transfer state ( $\sigma^{*}$ orbital located on the $\mathrm{NH}_{3}{ }^{+}$moiety) increases and opens or enhances other fragmentation channels. At even higher energies, the $\pi \sigma^{*}$ state is excited about $4000 \mathrm{~cm}^{-1}$ above the origin transition, leading to the formation of the radical cation after $\mathrm{H}$ loss from protonated tyramine. $^{22}$ In the case of protonated tyrosine, the same trend is observed, with a broad structure 
that appears about $6000 \mathrm{~cm}^{-1}$ above the band origin, which leads to the hydrogen loss channel $(\mathrm{m} / \mathrm{z}=181)$, the radical cation $\mathrm{m} / \mathrm{z} 181$ further fragmenting to $\mathrm{m} / \mathrm{z}=107 .^{38}$ In the case of protonated phenylethylamine and phenylalanine, the direct excitation of the $\pi \sigma^{*}$ state is expected to lie higher in energy. For phenylethylamine, the loss of $\mathrm{H}$ atom is observed $>6000 \mathrm{~cm}^{-1}$ above the band origin, ${ }^{22}$ while for phenylalanine, this channel cannot be reached with the available laser (Figure SI 2). As it will be detailed below, charge transfer states localized either on the ammonium or the carboxyl groups play a crucial role in the photodynamics of the protonated aromatic amino acids.

\section{Ab initio calculations}

\section{Comparison between spectroscopic results and ab initio calculations}

General considerations:

Well-resolved electronic photodissociation spectroscopy of protonated ions has made tremendous progress and it is thus worthwhile to compare with $a b$ initio calculations, which can shed light on the excited state relaxation processes. We have reported in Figure 3 the excited state energies calculated at the CC2/aug-cc-pVDZ level along with the molecular orbital (MO) representations at the first excited state optimized structures of the rot and stack conformers of $\mathrm{PheH}^{+}$and $\mathrm{TyrH}^{+}$, respectively. For sake of simplicity, we only present the results of the syn conformers of $\mathrm{TyrH}^{+}$.
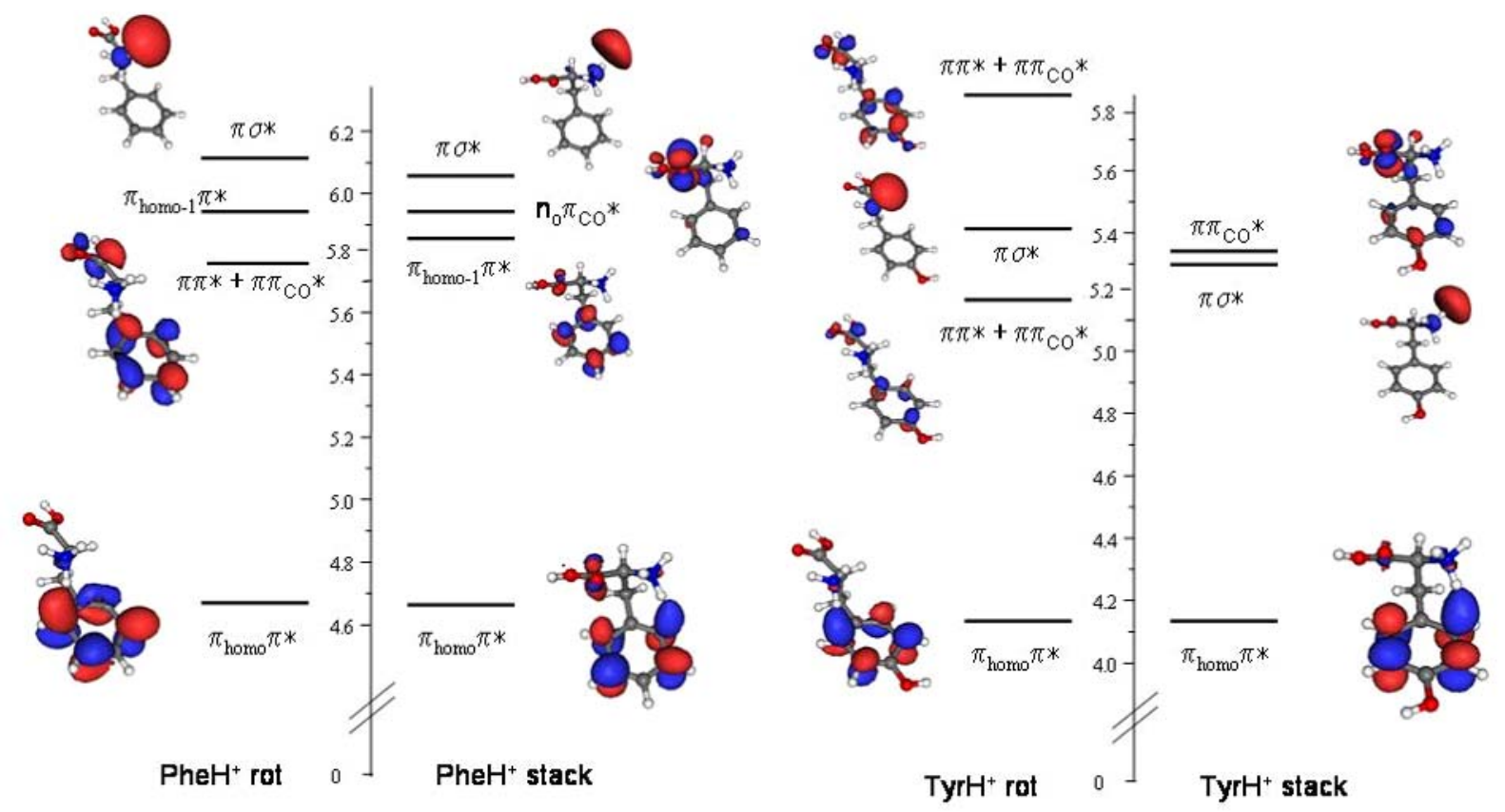

Figure 3: Excited state energies (CC2/aug-cc-pVDZ) along with the most contributing MO representation at the $S_{1}$ optimized structure for the rot and stack conformers of $\mathrm{PheH}^{+}$and $\mathrm{TyrH}^{+}$(syn orientation of the hydroxyl group). 
The electronic excited states of $\mathrm{PheH}^{+}$and $\mathrm{TyrH}^{+}$molecules share many similarities. The first excited state $S_{1}$ is noticeably separated from the higher excited states by roughly $1 \mathrm{eV}$. The electronic structure of $S_{1}$ is mainly of $\pi \pi^{*}$ character, corresponding to a pure $\pi_{\mathrm{HOMO}}-\pi^{*}$ transition $\left(\mathrm{L}_{\mathrm{b}}\right.$ state in the Platt's nomenclature ${ }^{49}$ ) in $\mathrm{TyrH}^{+}$, while in $\mathrm{PheH}^{+}$, there is a mixing between the $\pi_{\mathrm{HOMO}}-\pi^{*}$ and $\pi_{\mathrm{HOMO}-1}-\pi^{*}$ transitions along with a contribution of the $\pi_{\mathrm{CO}} *$ orbital. For both molecules, the optimized structure of the first excited state leads to a puckering of the aromatic ring and a lengthening of the $\mathrm{N}-\mathrm{H}$ bond pointing to the ring. This is also reflected in the mixing of the ring $\pi^{*}$ orbital on the carbon atom facing the ammonium group with the $3 p$ Rydberg ${ }^{34}$ orbital of the $\mathrm{NH}_{3}$ group. As it will be shown below, such structures can lead to the proton transfer reaction to the aromatic ring, as found in protonated tryptophan.

The electronic structures of the higher excited states are clearly different from the $S_{1}$ state and mostly involve diffuse electronic orbitals of $\sigma_{\mathrm{NH} 3} *$ and $\pi_{\mathrm{CO}} *$ characters. Because of the dense manifold of excited states from $\mathrm{S}_{2}$, it is not straightforward to firmly assign the electronic structures of these higher excited states. However, these excited states have a charge transfer character in which an electron from the aromatic ring is transferred to either $\mathrm{NH}_{3}{ }^{+}$or $\mathrm{CO}$ groups. In the last part of this section, we will show that the structure optimizations of $\pi \pi_{\mathrm{CO}}$ * states for both $\mathrm{PheH}^{+}$and $\mathrm{TyrH}^{+}$lead to proton transfer to the carboxylic group through a barrier, which is conformerdependent.

\section{$\pi \pi^{*}$ state: Franck-Condon (FC) analysis and proton transfer to the aromatic ring} FC analysis

In Table 1, we have reported the calculated energy of the optimized structures of the ground and first excited states (at the CC2/aug-cc-pVDZ level) for the two conformers of $\mathrm{PheH}^{+}$and the four conformers of $\mathrm{TyrH}^{+}$. The theoretical adiabatic $0_{0}{ }^{0}$ transition energy of all conformations has been calculated by taking into account the difference in zero point energy between the $S_{0}$ and the $S_{1}$ states. All the calculated $0_{0}{ }^{0}$ transition energies are within $\pm 500 \mathrm{~cm}^{-1}$ of the experimental values. For $\mathrm{PheH}^{+}$, the experimental origin transitions of the two conformers are very close, separated by only $9 \mathrm{~cm}^{-1}$, while the calculations predict a $31 \mathrm{~cm}^{-1}$ shift but of opposite sign. In $\mathrm{TyrH}^{+}$, the gap between the experimental band origins of the four conformers is larger and the calculations predict the same energy ordering between the transitions of the different conformers as the experiment. The electronic spectra simulated for the four conformers of TyrH + are plotted in Figure 4 and compared to the experimental spectrum recorded on m/z 108 in which all conformers are detected. All the calculated spectra are convoluted by a gaussian function of $3 \mathrm{~cm}^{-1}$ and the origin transitions calculated for each conformer are scaled to the experimental values, which allow predicting the first 
vibronic bands observed experimentally. For all conformers, the active modes involve motions of the $\mathrm{NH}_{3}{ }^{+}$group, the aromatic ring, the carboxylic acid group and out-of-plane bending of C-H group of the cycle (see Fig. SI 4).

Table 1: Energy (in $\mathrm{cm}^{-1}$ ) of the ground and first electronic states of the conformers of $\mathrm{PheH}^{+}$and $\mathrm{TyrH}^{+}$obtained by geometry optimization at the CC2/aug-cc-pVDZ level.

\begin{tabular}{lcccccc}
\hline Protonated amino acids & $\mathrm{S}_{0}$ & $\pi \pi^{*}$ adia. $^{\mathrm{a}}$ & $0_{0}{ }^{0}$ calc $^{\mathrm{b}}$ & $0_{0}{ }^{0} \exp ^{\mathrm{c}}$ & $\Delta^{\mathrm{exp}} /$ first $0_{0}{ }^{0 \text { (d) }}$ & $\Delta^{\text {calc(e) }}$ \\
\hline PheH+ stack & 0 & 39285 & 37912 & 37529 & 9 & -31 \\
PheH+ rot & 230 & 39298 & 37943 & 37520 & 0 & 0 \\
& & & & & & \\
TyrH+ stack syn & 0 & 35794 & 34493 & 35081 & 0 & 0 \\
TyrH+ stack anti & 42 & 35914 & 34619 & 35186 & 105 & 126 \\
TyrH+ rot syn & 260 & 35871 & 34584 & 35111 & 30 & 91 \\
TyrH+ rot anti & 265 & 36014 & 34738 & 35234 & 153 & 245 \\
\hline
\end{tabular}

\footnotetext{
(a) adiabatic $0_{0}^{0}$ energies calculated without zero point energy correction

${ }^{(b)} 0_{0}{ }^{0}$ calc energies are obtained after frequency calculations with correction for the difference in zero point energy between the ground and excited states.

${ }^{(\mathrm{c})} 0_{0}{ }^{0}$ exp represent the experimental transition origins.

${ }^{\text {(d) }} \Delta^{\exp } /$ first $0_{0}{ }^{0}$ represent the energy difference between the experimental transition origins, the reddest transition origin being the reference as in figure 1 and 2.

${ }^{(\mathrm{e})} \Delta^{\text {calc }}$ represent the energy difference between the calculated transition origins referenced to the experimental reddest transition origin.
}

For both molecules, intense vibronic bands are detected even at high excess energy above the origin transition. In $\mathrm{PheH}^{+}$, the $6 \mathrm{~b}$ (calculated at 514 and $512 \mathrm{~cm}^{-1}$ for the rot and stack conformers, respectively) and the 12 modes (calculated at $948 \mathrm{~cm}^{-1}$ ) in combination with the $6 \mathrm{~b}$ mode are observed along with the same progression of the low frequency modes built off of the origin transition. In $\mathrm{TyrH}^{+}$, the 1 mode (calculated at 812 and $804 \mathrm{~cm}^{-1}$ for the rot and stack (syn) conformers respectively) is observed along with the same low frequency vibrations as seen at the band origin. The $6 \mathrm{~b}$ and 12 modes have weak Franck-Condon activities and do not show up in the simulated FC spectra. As already mentioned in previous studies on toluene, ${ }^{46}$ aromatic amino $\operatorname{acids}^{4,12}$ and protonated aromatic amines, ${ }^{22}$ the large intensity of these bands arises from vibronic coupling between $\pi \pi^{*}$ excited states. In overall, the good agreement between the calculated band positions and the experimental transitions strongly supports that the electronic spectra of both molecules correspond to the $\pi \pi^{*}$ transition up to $2000 \mathrm{~cm}^{-1}$ above the origin band. 


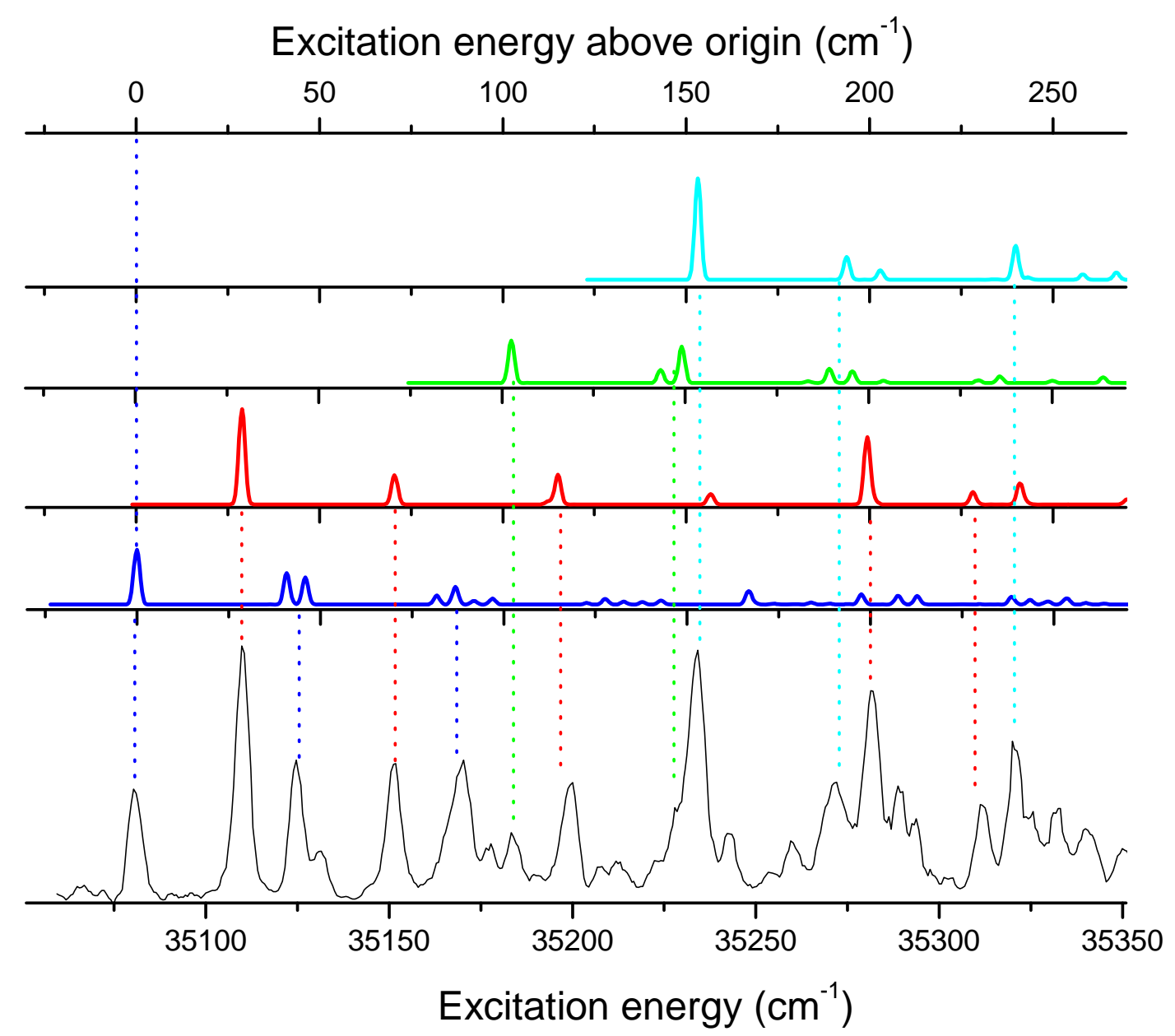

Figure 1: comparison between the experimental (recorded on the $\mathrm{m} / \mathrm{z} 108$, lower trace) and the calculated spectra (color on line: blue, stack/syn; red, rot/syn; green, stack/anti; cyan, rot/anti) of the 4 conformers of TyrH ${ }^{+}$.

\section{Minimum energy path for proton transfer to the ring}

In a previous theoretical paper, ${ }^{34}$ we have shown that proton transfer from the ammonium to the aromatic ring in $\mathrm{TrpH}^{+}$and $\mathrm{TyrH}^{+}$is a very efficient deactivation channel. In $\operatorname{TrpH} \mathrm{H}^{+}$, geometry optimization of the first excited state $S_{1}$ leads to a barrier-less proton transfer to the ring for all low lying conformers. These calculations agree with the very short excited state lifetime measured by pump-probe spectroscopy ${ }^{30}$ and corroborated by the UV photo-dissociation spectra recorded in the Rizzo's group. ${ }^{11}$ In $\mathrm{TyrH}^{+}$, geometry optimization of the $\mathrm{S}_{1}$ excited state induces the puckering of the aromatic ring with a ring dihedral angle of roughly $13^{\circ}$ and a lengthening of the $\mathrm{N}-\mathrm{H}$ bond pointing to the ring to $1.065 \AA$. The minimum energy path (MEP) for the proton transfer to the ring in $\mathrm{TyrH}^{+}$has already been calculated ${ }^{34}$ and exhibits a small energy barrier of $0.10 \mathrm{eV}$ at the CC2 level with a mixed basis sets (aug-cc-pVDZ for $\mathrm{N}$ and $\mathrm{O}$ atoms, SVP for $\mathrm{C}$ and $\mathrm{H}$ ). We have performed new calculations with the aug-cc-pVDZ basis set for all atoms and found an energy barrier of $0.15 \mathrm{eV}$ for the proton transfer to the ring for the stack and rot conformers of $\mathrm{TyrH}^{+}$syn. For PheH+, geometry optimization of the first excited state also induces a slight increase of the $\mathrm{NH}$ 
bond pointing to the ring and a puckering of the aromatic chromophore, but in a lesser extent than for $\mathrm{TyrH}^{+}$. The N-H bond increases to $1.049 \AA(1.027 \AA$ for the $\mathrm{N}-\mathrm{H}$ bond not involved in $\mathrm{H}$ bonding) and the ring dihedral angle shifts to $9^{\circ}$ instead of being planar (see Figure SI 5). As for $\mathrm{TyrH}^{+}$, we have calculated the MEP for the proton transfer to the ring by minimizing the first excited state energy at given N-H bond lengths. For both rot and stack conformers, we found an energy barrier of $0.25 \mathrm{eV}$ for the reaction, i.e. slightly larger than for Tyrosine. In Figure 5, we have plotted the MEP for proton transfer to the cycle as a function of the N-H bond length for the rot and stack conformers of $\mathrm{TyrH}^{+}$and $\mathrm{PheH}^{+}$. At N-H distance of $1.5 \AA$, the N-H constraint is released and the free optimization of the $\mathrm{S}_{1}$ leads to the proton transferred species (N-H distance around $\left.3.2 \AA\right)$ in which the ground and excited states are very close in energy. The proton transfer reaction in $\mathrm{S}_{1}$ is exothermic by about $0.6 \mathrm{eV}$ for $\mathrm{TyrH}^{+}$and $0.5 \mathrm{eV}$ for $\mathrm{PheH}^{+}$.
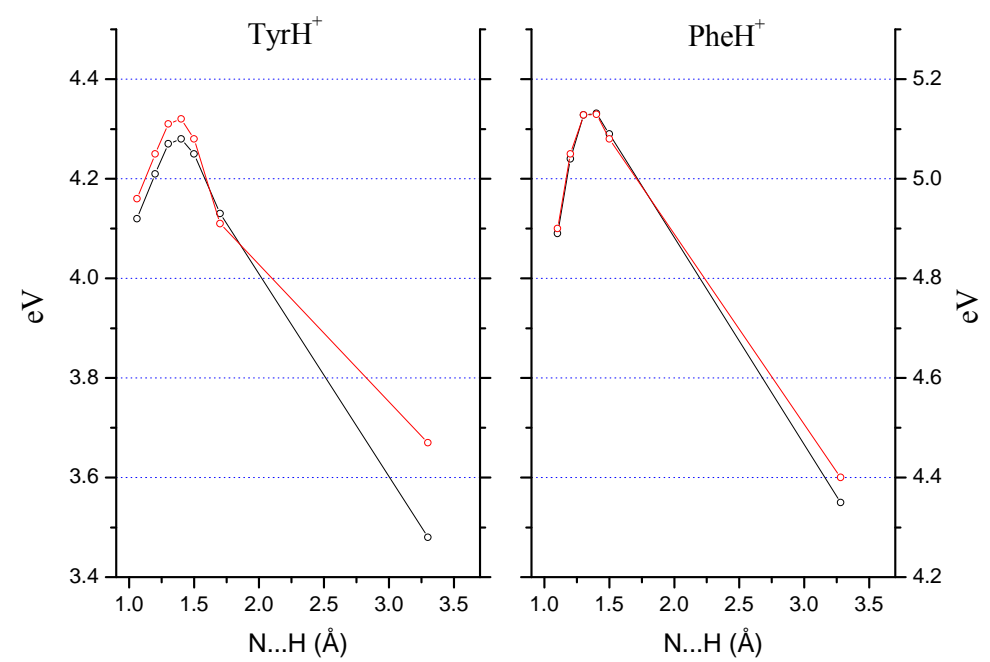

Figure 5: Minimum Energy Path (MEP) for the proton transfer reaction from the ammonium group to the aromatic ring for the stack (red line) and rot (black line) conformers of $\mathrm{TyrH}^{+}$syn (left) and $\mathrm{PheH}^{+}$(right). The last point at large $\mathrm{N}$ $H$ distance corresponds to the excited state energy of the transferred structures.

\section{$C_{\alpha}-C_{\beta}$ bond rupture after proton transfer to the ring: Natural Bond Orbitals (NBO) analysis}

We have performed a NBO analysis at the MP2/cc-pVDZ level for the rot and stack conformers of protonated tyrosine (syn) and protonated phenylalanine for the CC2/aug-cc-pVDZ optimized $\pi \pi^{*}$ structures and proton transferred to the cycle species. This analysis is conducted in order to reveal the destabilization of the $\mathrm{C}_{\alpha}-\mathrm{C}_{\beta}$ bond between the locally excited state structures $\left(\pi \pi^{*} \mathrm{~S}_{1}\right.$ state) and the structures where the proton has been transferred from the ammonium group to the aromatic cycle. The most important results are reported in Table SI1 (Supporting Information). It is noteworthy that the strength of the $\mathrm{C}_{\alpha}-\mathrm{C}_{\beta}$ bond is greatly reduced in the proton transferred species independently of the molecules or the conformations. This is reflected by the increase of the bond length (0.03-0.04 $\AA$ ) along with a drastic change in the electron density of the 
$\mathrm{C}_{\alpha}-\mathrm{C}_{\beta}$ bond. The occupation number of the $\mathrm{C}_{\alpha}-\mathrm{C}_{\beta}$ bond drops by $\sim 710^{-2}$ (atomic unit) in conjunction with the increase of its antibond of roughly $1.510^{-2}$ in $\mathrm{TyrH}^{+}$and $\mathrm{PheH}^{+}$. These changes have a noticeably high value and are by far the largest ones observed in the NBO analysis of the four calculated molecules. The NBO analysis (see Supporting Information) clearly emphasizes that the $\mathrm{C}_{\alpha}-\mathrm{C}_{\beta}$ bond is destabilized and explains why the protonated tyrosine and phenylalanine molecules dissociate through the $\mathrm{C}_{\alpha}-\mathrm{C}_{\beta}$ bond break following the proton transfer reaction to the cycle.

\section{Structure optimization of the higher electronic states}

Starting from the optimized structure of the $\pi \pi^{*} S_{1}$ state, we have optimized the structure of the $\pi \pi_{\mathrm{CO}} *$ excited states for the rot and stack conformers of $\mathrm{TyrH}^{+}$and $\mathrm{PheH}^{+}$. In both cases, we have followed the same procedure. A structure optimization is started on the electronic surface which has mainly a $\pi_{\mathrm{CO}} *$ character. When a crossing with the lower electronic state is reached, the optimization is stopped and started again on the $S_{n-1}$ surface, until the last crossing with the $S_{1}$ state occurs. At that point, structure optimization is performed on the $\mathrm{S}_{1}$ state, which has now acquired a $\pi \pi_{\mathrm{CO}} *$ character, until the convergence criteria are fulfilled or until the crossing with the ground electronic state is reached. Within this procedure, we can estimate the height of the barrier from the $\pi \pi^{*}$ to the $\pi \pi_{\mathrm{CO}} *$ states, which are reported in Figure 6 for the stack and rot conformers of $\mathrm{PheH}^{+}$ and $\mathrm{TyrH}^{+}$, respectively. Practically, for the stack conformers, the optimization starts from the $\mathrm{S}_{2}$ state, while for the rot conformer the optimization starts either from $\mathrm{S}_{3}$ or $\mathrm{S}_{4}$ states.

For both molecules, structure optimization of the $\pi \pi_{\mathrm{CO}} *$ state leads to a proton transfer reaction from the $\mathrm{NH}_{3}{ }^{+}$to the $\mathrm{CO}$ group. This excited state proton transfer reaction is exothermic by about $1 \mathrm{eV}$ and leads to a conical intersection with the ground electronic state. In the course of the $\pi \pi_{\mathrm{CO}} *$ state optimization, the locally excited $\pi \pi^{*}$ state $\left(\mathrm{S}_{1}\right.$ optimized structure) is destabilized, which leads to $\pi \pi^{*} / \pi \pi_{\mathrm{CO}} *$ barrier height that is conformer-dependent. In these transition state structures, there is a concomitant breaking of the planar symmetry of the carboxylic acid group (dihedral angle $\mathrm{OCOH}$ of $5^{\circ}$ ) and a slight lengthening of $0.01 \AA$ of the $\mathrm{N}-\mathrm{H}$ bond pointing to $\mathrm{CO}$, while the aromatic ring recovers planarity. For both molecules, the height of the barrier is in the order of 0.4-0.45 eV for the stack conformer and significantly larger (about 0.6-0.65 eV) for the rot structure. 

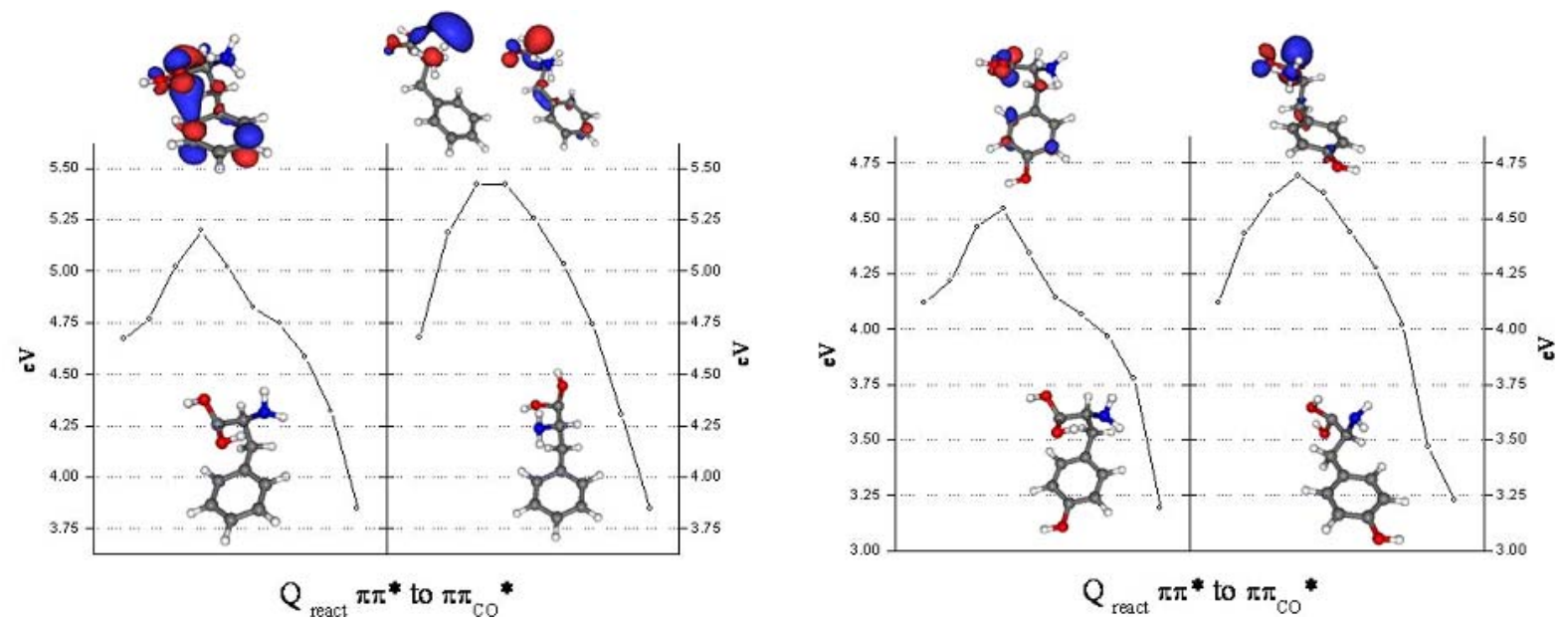

Figure 6: Potential energy profiles of the $S_{1}$ state for the proton transfer to CO reaction following the optimization of the $\pi \pi_{\mathrm{CO}}$ * excited state for the stack and rot conformers of $\mathrm{PheH}^{+}$(left) and $\mathrm{TyrH}^{+}$syn (right). The starting point is the adiabatic energy of the $\pi \pi^{*}$ state. The last point corresponds to the proton transferred species to the carboxylic group (structures represented at the bottom of the figures). For both molecules, the height of the barrier for the $\pi \pi^{*} / \pi \pi_{C O} *$ curve crossing is about $0.4 \mathrm{eV}$ for the stack conformer and $0.6 \mathrm{eV}$ for the rot structure. For each conformer, the most contributing molecular orbitals of the transition states are represented on the top of the figure.

In the stack conformer, electron transfer from the aromatic ring to the $\mathrm{CO}$ group is reflected by the $\pi^{*} / \pi_{\mathrm{CO}} *$ orbital overlap. This electron transfer triggers the proton transfer reaction to the oxygen atom of the $\mathrm{CO}$ group. This mechanism can thus be viewed as an electron-driven protontransfer process. In the rot conformer, the carboxylic acid group is not lying above the UV chromophore and direct electron transfer is hampered. However, during the excited state optimization, a concomitant stabilization and mixing of the $\sigma_{\mathrm{NH} 3} *$ and $\pi_{\mathrm{CO}} *$ orbitals first occurs. This mixing is evidenced by the most contributing molecular orbitals at the transition state structures of the rot conformers of $\mathrm{TyrH}^{+}$and $\mathrm{PheH}^{+}$depicted in Figure 6. When this transient point is reached, the excited state optimization is performed on the $S_{1}$ state and leads without barrier to the proton transfer reaction to the carbonyl with a pure $\pi_{\mathrm{CO}} *$ orbital on the carboxylic acid group.

\section{Discussion}

The electronic spectroscopy in $\mathrm{TyrH}^{+}$and $\mathrm{PheH}^{+}$is very similar. In the low frequency region around the band origin, Franck-Condon analysis confirms that the vibronic progression seen on the electronic spectra is assigned to low vibrational modes of the first $\pi \pi^{*}$ state of each conformer. The electronic spectra of these protonated molecules share many similarities with their neutral analogues, in particular, the large intensity of some modes induced by vibronic coupling with higher electronic excited states. Up to $2000 \mathrm{~cm}^{-1}$ above the band origin, the electronic spectra are thus firmly assigned to the excitation of the first $\pi \pi^{*}$ state.

The change in the fragmentation channels as a function of the excess energy in the $\pi \pi^{*}$ state is the most striking result. At the band origin, the main fragmentation channel corresponds to $\mathrm{m} / \mathrm{z}$ 
108 for $\mathrm{TyrH}^{+}$and m/z 92 and m/z 75 for $\mathrm{PheH}^{+}$. These fragments are produced through the $\mathrm{C}_{\alpha}-\mathrm{C}_{\beta}$ bond cleavage following proton transfer to the aromatic ring. Proton transfer reaction from the ammonium to the ring is indeed confirmed by $a b$ initio calculations. The optimized structures of the locally excited $\pi \pi^{*}$ state show ring puckering and lengthening of the N-H bond pointing to the ring. MEP reveals that this reaction has a low energy barrier of $0.15 \mathrm{eV}$ for protonated tyrosine and larger for protonated phenylalanine $(0.25 \mathrm{eV})$ and is independent of their conformations.

For $\mathrm{PheH}^{+}$, the $\mathrm{m} / \mathrm{z} 75$ fragment (glycine cation) is observed along with the $\mathrm{m} / \mathrm{z} 92$ fragment, and the former fragment is probably produced in the exit channel, via a back proton transfer when the two fragments separate. In $\mathrm{TyrH}^{+}$, only the $\mathrm{m} / \mathrm{z} 108$ fragment is detected. We tentatively rationalize this behavior by calculating, at the U-B3LYP/6-31+G* level of theory, the exothermicities of the $\mathrm{C}_{\alpha}-\mathrm{C}_{\beta}$ bond cleavage reaction leading to the two final ionic products, i.e. with the proton located on the glycine part or on the ring as indicated in Figure 7. The initial excess energy is determined by the energy of the $0_{0}{ }^{0}$ transition of the two protonated amino acids. For both protonated species, the most stable ionic fragments are m/z 92 and m/z 108 that correspond to the methylated aromatic cationic form. For $\mathrm{TyrH}^{+}$, calculations show that the exothermicity for the exit channel $\mathrm{m} / \mathrm{z} 75$ is negligible $(0.2 \mathrm{eV})$ if compared to the reaction leading to the $\mathrm{m} / \mathrm{z} 108$ fragment. For $\mathrm{PheH}+$, the exit channel m/z 75 is open with the available energy in the system, which could explain the observation of both $\mathrm{m} / \mathrm{z} 75$ and $\mathrm{m} / \mathrm{z} 92$.

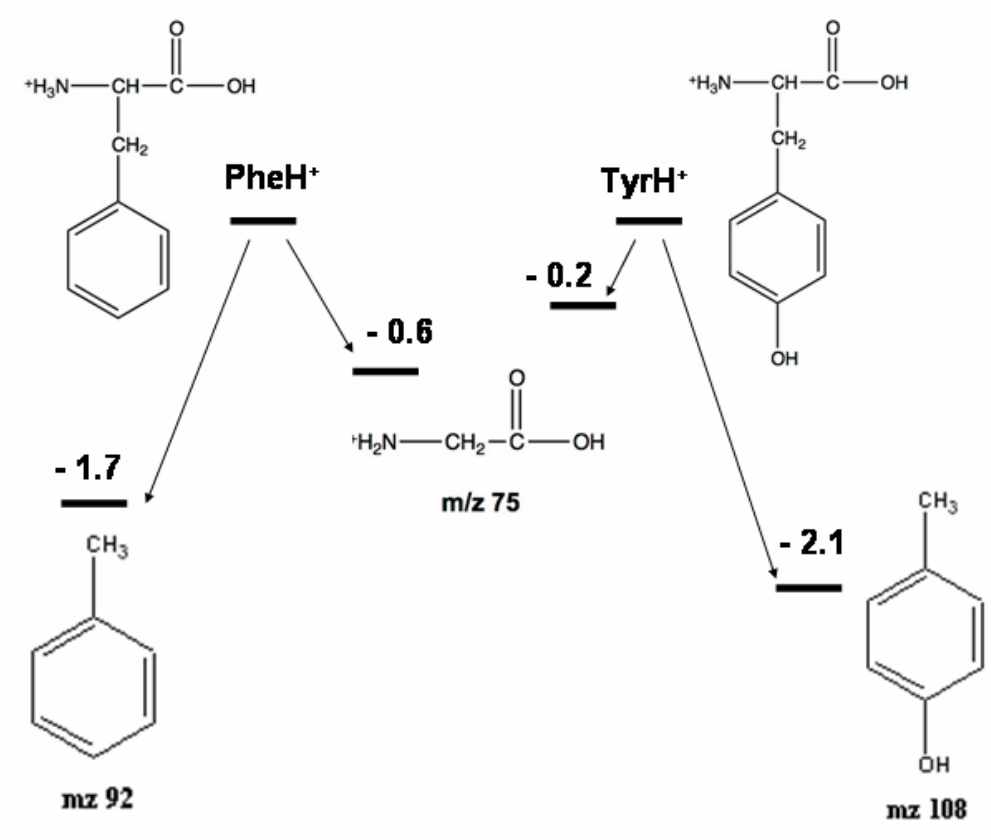

Figure 7: Calculated exothermicities (energies in $\mathrm{eV}$ ) of $C_{\alpha}$ - $C_{\beta}$ bond cleavage reactions leading to the final ionic products in $\mathrm{PheH}+$ and $\mathrm{TyrH}+$. 
The $\mathrm{C}_{\alpha}-\mathrm{C}_{\beta}$ bond cleavage reaction is in competition with a second reaction leading to the detection of new ionic fragments related to the loss of neutral $\mathrm{H}_{2} \mathrm{O}+\mathrm{CO}$. This reaction seems to be correlated to the coupling of the locally excited $\pi \pi^{*}$ state with higher excited states since it appears at higher excitation energy. Indeed, dynamics on the $\pi \pi_{\mathrm{CO}} *$ state leads without barrier to proton transfer to the carbonyl group. The locally excited $\pi \pi^{*}$ state is decaying through this channel via $\pi \pi^{*} / \pi \pi_{\mathrm{CO}} *$ curve crossing, which is located higher in energy than the barrier for proton transfer to the aromatic ring. This process becomes the dominant deactivation channel from roughly $1000 \mathrm{~cm}^{-1}$ above the transition origin. It is noteworthy that the two deactivation processes are not really in competition as soon as the $\pi \pi_{\mathrm{CO}} *$ state is accessible. This can be explained by the nature of the driving force for the two proton transfer reactions. In the case of proton transfer to the ring, reaction involves also large atomic displacements such as ring puckering, while the electronic density stays localized on the aromatic ring. At the opposite, reaching the $\pi \pi_{\mathrm{CO}} *$ state from the locally excited $\pi \pi^{*}$ state implies an electron transfer from the ring to the carboxylic acid group, which drives very efficiently and without barrier the proton transfer from the ammonium. In the case of $\mathrm{PheH}^{+}$, the proton transfer reaction to the cycle (energy barrier of $0.25 \mathrm{eV}$ ) is no more competitive from 800 $\mathrm{cm}^{-1}$ of excess energy in the $\pi \pi^{*}$ state. Conversely, in $\mathrm{TyrH}^{+}$, the proton transfer reaction to the ring above $800 \mathrm{~cm}^{-1}$ is still feasible due to the lower energy barrier $(0.15 \mathrm{eV})$ but is conformerdependent.

$A b$ initio calculations reveal that the height of the barrier between the $\pi \pi *$ locally excited state and the $\pi \pi_{\mathrm{CO}} *$ state is conformer-dependent. Stack conformers, in which the carboxylic acid group lies above the aromatic ring, have lower energy barrier $(0.4 \mathrm{eV})$ than the rot conformers (barrier of $0.6 \mathrm{eV}$ ). This is consistent with the high conformer selectivity experimentally observed in $\mathrm{TyrH}^{+}$in which rot conformations have the most intense fragmentation yield at the origin transition and are still detected from $800 \mathrm{~cm}^{-1}$ of excess energy on the $\mathrm{m} / \mathrm{z} 108$ channel. This implies that the proton transfer reaction to the ring (barrier of $0.15 \mathrm{eV}$ ) for the rot conformers is in competition with the proton transfer reaction to the carbonyl (barrier of $0.6 \mathrm{eV}$ ). In contrast, the stack conformations are mostly detected on the m/z 147, m/z 136, m/z 123 and m/z 119 channels around the origin transition and exclusively from $800 \mathrm{~cm}^{-1}$ of excess energy. These fragmentation channels are thus assigned to the access and dynamics on the $\pi \pi_{\mathrm{CO}} *$ state. 

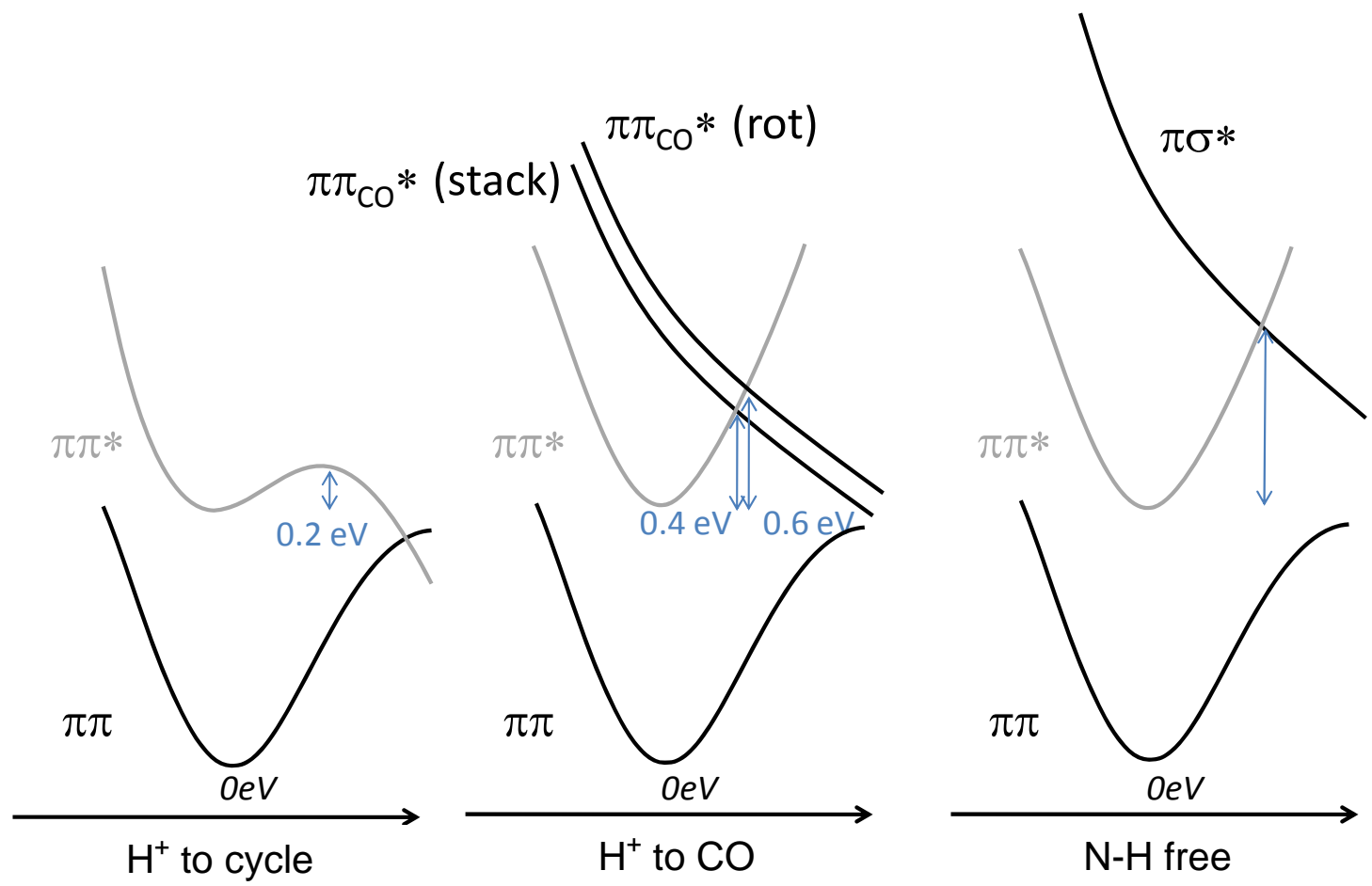

Figure 8: Schematic potential energy functions for the excited states of $\mathrm{PheH}^{+}$or $\mathrm{TyrH}^{+}$as a function of the proton transfer coordinate towards the aromatic ring (left), towards $\mathrm{CO}$ (middle), and versus the $\mathrm{NH}_{\text {free }}$ coordinate (right). The barrier heights are qualitatively indicated by blue arrows. They differ from one conformer to the other along the proton transfer to the CO.

To sum up, the different photo-fragmentation channels can be inferred from the geometry of the protonated amino acids in their excited states. Indeed, one hydrogen atom of the protonated amino group is pointing toward the ring (inducing a strong puckering of the chromophore), another one to the carbonyl and the last one is "free". Following excitation of the first electronic state $\left(\pi \pi^{*}\right)$ and because of its coupling with other electronic states $\left(\pi \pi_{\mathrm{CO}}^{*}\right.$ and $\left.\pi \sigma^{*}\right)$, several proton transfer or bond dissociations can occur (Figure 8). Excitation of the $\pi \pi^{*}$ state leads to the proton transfer to the aromatic ring and to $\mathrm{C}_{\alpha}-\mathrm{C}_{\beta}$ bond cleavage. This channel is in competition with a second channel: the proton transfer from the $\mathrm{NH}_{3}{ }^{+}$to the carbonyl, which is due to a crossing between the $\pi \pi^{*}$ state and the $\pi \pi_{\mathrm{CO}} *$ giving the $\mathrm{H}_{2} \mathrm{O}+\mathrm{CO}$ loss $\left(\mathrm{m} / \mathrm{z} 136\right.$ and $\mathrm{m} / \mathrm{z} 120$ for $\mathrm{TyrH}^{+}$and $\mathrm{PheH}^{+}$, respectively). The third channel ( $\mathrm{NH}$ dissociation) has been observed at higher excitation energies for $\mathrm{TyrH}^{+}{ }^{38}$

The UV induced photo-fragmentation of the protonated amines ${ }^{22}$ and amino acids shows drastic competitions in the fragmentation channels as a function of the excess energy in the locally excited state. In both cases, the coupling of the $\pi \pi^{*}$ state with charge transfer states plays a key role and triggers proton transfer reactions from the ammonium to different acceptor groups. In protonated amines, one hydrogen of the ammonium group is involved in a strong $\mathrm{H}$-bond 
interaction with the aromatic chromophore while in the protonated amino acids, the ammonium group faces both the aromatic ring and the carboxylic acid group, leading to a competition in the proton transfer reactions. In all cases, the electronic spectra are assigned to $\pi \pi^{*}$ transition. In the low energy region, the main fragmentation channel corresponds to the $C_{\alpha}-C_{\beta}$ bond cleavage following proton transfer to the ring. In the course of proton transfer reaction, the electronic density stays localized on the aromatic ring, with large nuclear displacements. The coupling between the locally excited state and charge transfer states increases with the excess energy and triggers the opening of new fragmentation channels. For protonated phenylethylamine and tyramine, the only charge transfer state present has a $\sigma^{*}$ electronic character in which an electron from the $\pi^{*}$ orbital on the aromatic ring is promoted to a $\sigma^{*}$ Rydberg like orbital localized on the $\mathrm{NH}_{3}{ }^{+}$group. The $\pi \pi^{*} / \pi \sigma^{*}$ dynamic is responsible for the opening of the $\mathrm{NH}_{3}$ loss channel as the excess energy above the $0_{0}{ }^{0}$ transition increases. In the case of the protonated amino acids, two low-lying charge transfer states ( $\pi \pi_{\mathrm{CO}} *$ and $\pi \sigma^{*}$ states) can couple with the $\pi \pi *$ and it is not straightforward to reveal which of these states triggers the excited state dynamics leading to the opening of the new fragmentation channels. However, the fragmentation properties of the stack conformers are clearly different from the rot structures, which are supported by the theoretical calculations on the $\pi \pi_{\mathrm{CO}} *$ excited state. The coupling between the locally excited state and charge transfer states depends on the overlap of the molecular orbitals. Irrespective of the conformers, the ammonium group lies above the aromatic ring which ensure a constant $\pi \pi^{*} / \pi \sigma^{*}$ coupling and no conformer selectivity. In contrast, the carboxylic group lies above the aromatic ring only for the stack conformers, which would explain the observed conformational selectivity.

\section{Conclusions}

These new spectroscopic experiments and calculations on protonated tyrosine and phenylalanine give clear evidence of the extreme dependence of the UV induced fragmentation channels upon the electronic nature of the electronic excited states. The vibrational analysis of the electronic spectra of both molecules shows that the locally excited state has a $\pi \pi^{*}$ character up to $2000 \mathrm{~cm}^{-1}$ above the band origin. At the band origin, the main fragmentation channel corresponds to the $\mathrm{C}_{\alpha}-\mathrm{C}_{\beta}$ bond cleavage following proton transfer from the ammonium group to the aromatic ring. This fragmentation channel is specific to the electronic excitation in the $S_{1}$ state and is not observed in the collision induced dissociation experiment. The calculated energy barrier for this reaction is in the order of $0.2 \mathrm{eV}$ for both molecules. As the excess energy in the $\pi \pi^{*}$ state increases, new fragmentation channels related to the loss of $\mathrm{CO}+\mathrm{H}_{2} \mathrm{O}$ open and compete with the $\mathrm{C}_{\alpha}-\mathrm{C}_{\beta}$ bond cleavage reaction. For both molecules, these new fragmentation channels are triggered by a 
coupling of the locally excited state with the $\pi \pi_{\mathrm{CO}} *$ state leading to a proton transfer from the ammonium to the carboxyl group. The charge transfer from the aromatic ring to the carboxylic acid group depends on the overlap of the corresponding orbitals and is thus conformer-dependent, as experimentally observed. The stack conformers, in which the carboxylic acid group lies above the aromatic ring, have a lower excited state energy barrier (about $0.4 \mathrm{eV}$ ) for the proton transfer reaction to the carbonyl than the rot conformations (about $0.6 \mathrm{eV}$ ). We are currently investigated the excited state lifetimes of the different conformers of these molecules as a function of the excess energy in the $\pi \pi^{*}$ state through a pump/probe detection scheme at picosecond resolution in order to reveal the excited state dynamics and coupling in these protonated aromatic amino acids.

\section{Acknowledgements}

This work has been supported by the Université Paris-Sud 11, by the ANR research Grant (ANR2010BLANC040501), the RTRA “Triangle de la Physique" COMOVA and COMOVA II. We acknowledge the mechanical staff, especially Eric Bouisset and Jérome Guigand for machining parts of the experimental setup, and Christophe Charrière for the electronics. We also acknowledge Valerie Brenner for helpful discussions. We acknowledge the uses of the computing facility clusters GMPCS of the LUMAT federation (FR LUMAT 2764) and MAGI of the University Paris 13.

\section{Supporting Information Available}

Photo-fragmentation mass spectrum of $\mathrm{PheH}^{+}$; Photo-fragmentation spectra of $\mathrm{PheH}^{+}$up to 7000 $\mathrm{cm}^{-1}$ above the origin transition; Laser power dependence of the fragmentation yield in $\mathrm{TyrH}^{+}$; Details of the Franck-Condon simulation of $\mathrm{TyrH}^{+}$rot conformer; $\mathrm{S}_{1}$ optimized structures of $\mathrm{TyrH}^{+}$ and $\mathrm{PheH}^{+}$; Details of the Natural Bond Orbitals (NBO) analysis. This information is available free of charge via the Internet at http://pubs.acs.org.

\section{References}

(1) Cohen, R.; Brauer, B.; Nir, E.; Grace, L.; de Vries, M. S. Resonance-Enhanced Multiphoton Ionization Spectroscopy of Dipeptides. J. Phys. Chem. A 2000, 104, 6351-6355.

(2) Snoek, L. C.; Robertson, E. G.; Kroemer, R. T.; Simons, J. P. Conformational Landscapes in Amino Acids: Infrared and Ultraviolet Ion-Dip Spectroscopy of Phenylalanine in the Gas Phase. Chem. Phys. Lett. 2000, 321, 49-56.

(3) Martinez III, S.; Alfano, J.; Levy, D. The Electronic Spectroscopy of the Amino Acids Tyrosine and Phenylalanine in a Supersonic Jet. J. Mol. Spectrosc. 1992, 430, 421-430.

(4) Grace, L. I.; Cohen, R.; Dunn, T. .; Lubman, D. M.; de Vries, M. S. The R2PI Spectroscopy of Tyrosine: A Vibronic Analysis. J. Mol. Spectrosc. 2002, 215, 204-219. 
(5) Shimozono, Y.; Yamada, K.; Ishiuchi, S.; Tsukiyama, K.; Fujii, M. Revised Conformational Assignments and Conformational Evolution of Tyrosine by Laser Desorption Supersonic Jet Laser Spectroscopy. Phys. Chem. Chem. Phys. 2013, 15, 5163-5175.

(6) Inokuchi, Y.; Kobayashi, Y.; Ito, T.; Ebata, T. Conformation of L-Tyrosine Studied by FluorescenceDetected UV-UV and IR-UV Double-Resonace Spectroscopy. J. Phys. Chem. A 2007, 111, 32093215.

(7) Rizzo, T. R.; Park, Y. D.; Peteanu, L. A.; Levy, D. H. The Electronic Spectrum of the Amino Acid Tryptophan in the Gas Phase. J. Chem. Phys. 1986, 84, 2534.

(8) Snoek, L. C.; Kroemer, R. T.; Hockridge, M. R.; Simons, J. P. Conformational Landscapes of Aromatic Amino Acids in the Gas Phase: Infrared and Ultraviolet Ion Dip Spectroscopy of Tryptophan. Phys. Chem. Chem. Phys. 2001, 3, 1819-1826.

(9) Bakker, J. M.; Mac Aleese, L.; Meijer, G.; von Helden, G. Fingerprint IR Spectroscopy to Probe Amino Acid Conformations in the Gas Phase. Phys. Rev. Lett. 2003, 91, 203003.

(10) Talbot, F. O.; Tabarin, T.; Antoine, R.; Broyer, M.; Dugourd, P. Photodissociation Spectroscopy of Trapped Protonated Tryptophan. J. Chem. Phys. 2005, 122, 074310.

(11) Boyarkin, O. V; Mercier, S. R.; Kamariotis, A.; Rizzo, T. R. Electronic Spectroscopy of Cold Protonated Tryptophan and Tyrosine. J. Am. Chem. Soc. 2006, 128, 2816-2817.

(12) Stearns, J. A.; Mercier, S.; Seaiby, C.; Guidi, M.; Boyarkin, O. V; Rizzo, T. R. ConformationSpecific Spectroscopy and Photodissociation of Cold, Protonated Tyrosine and Phenylalanine. J. Am. Chem. Soc. 2007, 129, 11814-11820.

(13) Mercier, S. R.; Boyarkin, O. V; Kamariotis, A.; Guglielmi, M.; Tavernelli, I.; Cascella, M.; Rothlisberger, U.; Rizzo, T. R. Microsolvation Effects on the Excited-State Dynamics of Protonated Tryptophan. J. Am. Chem. Soc. 2006, 128, 16938-16943.

(14) Wang, Y. S.; Tsai, C. H.; Lee, Y. T.; Chang, H. C.; Jiang, J. C.; Asvany, O.; Schlemmer, S.; Gerlich, D. Investigations of Protonated and Deprotonated Water Clusters Using a Low-Temperature 22-Pole Ion Trap. J. Phys. Chem. A 2003, 107, 4217-4225.

(15) Fujihara, A.; Matsumoto, H.; Shibata, Y.; Ishikawa, H.; Fuke, K. Photodissociation and Spectroscopic Study of Cold Protonated Dipeptides. J. Phys. Chem. A 2008, 112, 1457-1463.

(16) Fujihara, A.; Noguchi, N.; Yamada, Y.; Ishikawa, H.; Fuke, K. Microsolvation and Protonation Effects on Geometric and Electronic Structures of Tryptophan and Tryptophan-Containing Dipeptides. J. Phys. Chem. A 2009, 113, 8169-8175.

(17) Redwine, J. G.; Davis, Z. A.; Burke, N. L.; Oglesbee, R. A.; McLuckey, S. A.; Zwier, T. S. A Novel Ion Trap Based Tandem Mass Spectrometer for the Spectroscopic Study of Cold Gas Phase Polyatomic Ions. Int. J. Mass Spectrom. 2013, 348, 9-14.

(18) Wang, X.-B.; Wang, L.-S. Development of a Low-Temperature Photoelectron Spectroscopy Instrument Using an Electrospray Ion Source and a Cryogenically Controlled Ion Trap. Rev. Sci. Instrum. 2008, 79, 073108.

(19) Kamrath, M. Z.; Relph, R. A.; Guasco, T. L.; Leavitt, C. M.; Johnson, M. A. Vibrational Predissociation Spectroscopy of the H2-Tagged Mono- and Dicarboxylate Anions of Dodecanedioic Acid. Int. J. Mass Spectrom. 2011, 300, 91-98. 
(20) Choi, C. M.; Choi, D. H.; Kim, N. J.; Heo, J. Effective Temperature of Protonated Tyrosine Ions in a Cold Quadrupole Ion Trap. Int. J. Mass Spectrom. 2012, 314, 18-21.

(21) Alata, I.; Bert, J.; Broquier, M.; Dedonder, C.; Feraud, G.; Grégoire, G.; Soorkia, S.; Marceca, E.; Jouvet, C. Electronic Spectra of the Protonated Indole Chromophore in the Gas Phase. J. Phys. Chem. A 2013, 117, 4420-4427.

(22) Féraud, G.; Broquier, M.; Dedonder-Lardeux, C.; Grégoire, G.; Soorkia, S.; Jouvet, C. Photofragmentation Spectroscopy of Cold Protonated Aromatic Amines in the Gas Phase. Phys. Chem. Chem. Phys. 2014, 16, 5250-5259.

(23) El Aribi, H.; Orlova, G.; Hopkinson, A. C.; Siu, K. W. M. Gas-Phase Fragmentation Reactions of Protonated Aromatic Amino Acids: Concomitant and Consecutive Neutral Eliminations and Radical Cation Formations. J. Phys. Chem. A 2004, 108, 3844-3853.

(24) Svendsen, A.; Lorenz, U. J.; Boyarkin, O. V; Rizzo, T. R. A New Tandem Mass Spectrometer for Photofragment Spectroscopy of Cold, Gas-Phase Molecular Ions. Rev. Sci. Instrum. 2010, 81, 073107.

(25) Lepère, V.; Lucas, B.; Barat, M.; Fayeton, J. A.; Picard, V. J.; Jouvet, C.; Carcabal, P.; Nielsen, I.; Dedonder-Lardeux, C.; Grégoire, G.; et al. Comprehensive Characterization of the Photodissociation Pathways of Protonated Tryptophan. J. Chem. Phys. 2007, 127, 134313.

(26) Lepère, V.; Lucas, B.; Barat, M.; Fayeton, J. A.; Picard, Y. J.; Jouvet, C.; Carcabal, P.; Nielsen, I. B.; Dedonder-Lardeux, C.; Grégoire, G.; et al. Characterization of Neutral Fragments Issued from the Photodissociation of Protonated Tryptophane. Phys. Chem. Chem. Phys. 2007, 9, 5330-5334.

(27) Lucas, B.; Barat, M.; Fayeton, J. A.; Perot, M.; Jouvet, C.; Grégoire, G.; Brøndsted Nielsen, S. Mechanisms of Photoinduced C-Alpha-C-Beta Bond Breakage in Protonated Aromatic Amino Acids. J. Chem. Phys. 2008, 128, 164302.

(28) Grégoire, G.; Lucas, B.; Barat, M.; Fayeton, J. A.; Dedonder-Lardeux, C.; Jouvet, C. UV Photoinduced Dynamics in Protonated Aromatic Amino Acid. Eur. Phys. J. D 2009, 51, 109-116.

(29) Kang, H.; Jouvet, C.; Dedonder-Lardeux, C.; Martrenchard, S.; Grégoire, G.; Desfrançois, C.; Schermann, J.-P.; Barat, M.; Fayeton, J. A. Ultrafast Deactivation Mechanisms of Protonated Aromatic Amino Acids Following UV Excitation. Phys. Chem. Chem. Phys. 2005, 7, 394-398.

(30) Kang, H.; Dedonder-Lardeux, C.; Jouvet, C.; Grégoire, G.; Desfrançois, C.; Schermann, J.-P.; Barat, M.; Fayeton, J. A. Control of Bond-Cleaving Reactions of Free Protonated Tryptophan Ion by Femtosecond Laser Pulses. J. Phys. Chem. A 2005, 109, 2417-2420.

(31) Serrano-Andres, L.; Roos, B. O. Theoretical Study of the Absorption and Emission Spectra of Indole in the Gas Phase and in a Solvent. J. Am. Chem. Soc. 1996, 25, 185-195.

(32) Gindensperger, E.; Haegy, A.; Daniel, C.; Marquardt, R. Ab Initio Study of the Electronic Singlet Excited-State Properties of Tryptophan in the Gas Phase: The Role of Alanyl Side-Chain Conformations. Chem. Phys. 2010, 374, 104-110.

(33) Blancafort, L.; González, D.; Olivucci, M.; Robb, M. A. Quenching of Tryptophan $1\left(\pi, \pi^{*}\right)$ Fluorescence Induced by Intramolecular Hydrogen Abstraction via an Aborted Decarboxylation Mechanism. J. Am. Chem. Soc. 2002, 124, 6398-6406. 
(34) Grégoire, G.; Jouvet, C.; Dedonder, C.; Sobolewski, A. L. Ab Initio Study of the Excited-State Deactivation Pathways of Protonated Tryptophan and Tyrosine. J. Am. Chem. Soc. 2007, 129, $6223-$ 6231.

(35) Grégoire, G.; Jouvet, C.; Dedonder, C.; Sobolewski, A. L. On the Role of Dissociative $(\pi, \mathrm{s})^{*}$ States in the Photochemistry of Protonated Tryptamine and Tryptophan: An Ab Initio Study. Chem. Phys. 2006, 324, 398-404.

(36) Nolting, D.; Marian, C.; Weinkauf, R. Protonation Effect on the Electronic Spectrum of Tryptophan in the Gas Phase. Phys. Chem. Chem. Phys. 2004, 6, 2633-2640.

(37) Lakowicz, J. R. Principles of Fluorescence Spectroscopy; 2nd ed.; Springer: New York, 1999.

(38) Dedonder, C.; Féraud, G.; Jouvet, C. Photophysics of Ionic Biochromophores; Brøndsted Nielsen, S.; Wyer, J. A., Eds.; Springer-Verlag Berlin and Heidelberg GmbH and Co., 2013; pp. 155-180.

(39) Andersen, L. H.; Lapierre, A.; Nielsen, S. B.; Nielsen, I. B.; Pedersen, S. U.; Pedersen, U. V; Tomita, S. Chromophores of the Green Fluorescent Protein Studied in the Gas Phase Eur. Phys. J. D 2002, 20, 597-600.

(40) Ahlrichs, R.; Bär, M.; Häser, M.; Horn, H.; Kölmel, C. Electronic Structure Calculations on Workstation Computers: The Program System Turbomole. Chem. Phys. Lett. 1989, 162, 165-169.

(41) Weigend, F.; Häser, M. RI-MP2: First Derivatives and Global Consistency. Theor. Chem. Acc. 1997, 97, 331-340.

(42) Dunning, T. H. Gaussian Basis Sets for Use in Correlated Molecular Calculations. I. The Atoms Boron through Neon and Hydrogen. J. Chem. Phys. 1989, 90, 1007.

(43) Western, C. M. PGOPHER, a Program for Simulating Rotational Structure; University of Bristol: Bristol, U.K., 2010; available at http:// pgopher.chm.bris.ac.uk.

(44) Glendening, E. D.; Reed, A. E.; Carpenter, J. E.; Weinhold, F. NBO Version 3.1.

(45) Frisch, M. J.; Trucks, G. W.; Schlegel, H. B.; Scuseria, G. E.; Robb, M. A.; Cheeseman, J. R.; Scalmani, G.; Barone, V.; Mennucci, B.; Petersson, G. A. et al. Gaussian 09, Revision A.02, Gaussian, Inc., Wallingford CT, 2009.

(46) Hopkins, J. B.; Powers, D. E.; Smalley, R. E. Vibrational Relaxation in Jet-Cooled Alkyl Benzenes. I.Absorption Spectra. J. Chem. Phys. 1980, 72, 5039.

(47) Sun, S.; Bernstein, E. R. Aromatic van Der Waals Clusters: Structure and Nonrigidity. J. Phys. Chem. 1996, 100, 13348-13366.

(48) Sobolewski, A. L.; Domcke, W.; Dedonder-Lardeux, C.; Jouvet, C. Excited-State Hydrogen Detachment and Hydrogen-Transfer Driven by Repulsive $\pi \sigma^{*}$ States: A New Paradigm for Non Radiative Decay in Biomolecules. Phys. Chem. Chem. Phys. 2002, 4, 1093-1100.

(49) Platt, J. R. Classification of Spectra of Cata-Condensed Hydrocarbons. J. Chem. Phys. 1949, 17, 484. 
« Table of Contents » Graphic :

\section{Protonated Phenylalanine}

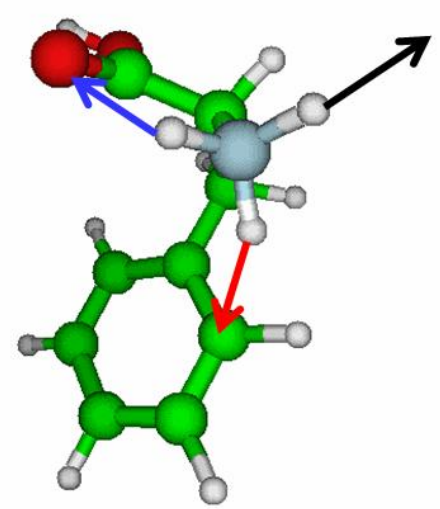

3 Hydrogen = 3 fragmentation pathways 